\title{
How to balance the treatment of stress urinary incontinence among female athletes?
}

Paweł Rzymski ${ }^{1}$, Bartłomiej Burzyński², Michalina Knapik ${ }^{3}$, Jacek Kociszewski ${ }^{4}$, Maciej Wilczak ${ }^{1}$

\author{
'Department of Mother's and Child's Health, Poznań, Poland \\ 2Department of Rehabilitation, School of Health Science, Medical University of Silesia, \\ Katowice, Poland \\ ${ }^{3}$ Faculty of Medical Sciences in Zabrze, Medical University of Silesia in Katowice, Poland \\ ${ }^{4}$ Department of Gynecology and Obstetrics, Evangelical Hospital Hagen-Haspe, \\ Hagen, Germany
}

Submitted: 14 July 2020; Accepted: 1 October 2020;

Online publication: 21 October 2020

Arch Med Sci 2021; 17 (2): 314-322

DOI: https://doi.org/10.5114/aoms.2020.100139

Copyright @ 2020 Termedia \& Banach

\author{
Corresponding author: \\ Paweł Rzymski \\ Department of Mother's \\ and Child's Health \\ 33 Polna St \\ 60-535 Poznań, Poland \\ E-mail: \\ parzymsk@gpsk.am.poznan.pl
}

\begin{abstract}
Urinary incontinence in the general population occurs in $7 \%$ of nonpregnant women under 39 years old, $17 \%$ of those 40 to 59 years old, and $23-32 \%$ of those over 60 years old. In athletes the prevalence is higher, especially in high-impact training and gravity sports. Pelvic floor muscles (PFM) have two important roles; they serve as the support for abdominal organs and are crucial for closure of the urethra, vagina and rectum. We present the proper mechanisms of PFM caudal contractions with proper abdominal muscle control to avoid excessive intra-abdominal pressure. Pelvic floor sonography is discussed as the only objective method for pelvic floor examination among sportswomen and a tool which should be used routinely by urophysiotherapists and urogynecologists. A multidisciplinary individualized approach to stress urinary incontinence among athletes is presented including: physiotherapy, diagnostic imaging, use of a pessary, tampons, pharmacologic and surgical treatment. We present guidelines for stress urinary incontinence treatment in sportswomen of different age.
\end{abstract}

Key words: physiotherapy, athletes, diagnostic imaging, urinary incontinence, high impact training.

\section{Introduction}

Urinary incontinence (UI) is a common condition which worsens the quality of life among women. It is defined as any involuntary urine leakage and it is more prevalent in older women. One type is stress urinary incontinence (SUI), which can occur separately or mixed with urge symptoms. Stress urinary incontinence is defined as the "involuntary loss of urine on effort or physical exertion, or on sneezing or coughing". According to the studies the prevalence varies between $3 \%$ and $58 \%$ and is caused by different criteria, age, populations, and recruitment to the studies [1-5]. A higher prevalence of up to $80 \%$ has also been reported [6]. Repetitive, high-impact sports pose an obvious risk for UI, but urinary complaints are under-reported, under-recognized and under-treated [7]. The age-dependent prevalence of $U I$ in the general population has been described as follows: $7 \%$ in non-pregnant women under 39 years, $17 \%$ in women aged 40 to 59 years, and $23-32 \%$ of those aged 60 years 
or over. During pregnancy the risk is higher, but all the statistics are also underestimated [4, 8]. Stress urinary incontinence is also a dominating symptom among triathletes, although swimming and cycling are considered to be less risky than running. However, this particular accumulation of three disciplines with high-volume and cross training probably produces a synergic negative effect on the pelvic floor. The occurrence of SUI was found to be $37.4 \%$ among sportswomen preparing for competition up to ironman distance (cumulative 140.6 miles) [9]. Dos Santos et al. quantified urine loss using a pad test to illustrate the differences between high and low impact disciplines. Running, skating, basketball and karate were at higher risk, whereas cycling, ballet and contemporary dance were all negative in pad tests. Cumulative training experience over years, and earlier commencement of exercise were an additional two factors correlated with SUI. Interestingly $24 \%$ of patients who considered themselves as continent were also positive in the pad test; thus the authors diagnosed them with SUI [5].

Moderate physical activity does not seem to increase the risk for UI, but strenuous exercise should be considered as a risk factor. Thus, the discipline determines its occurrence; some of the highest rates have been reported among gymnasts, trampolinists and athletes [2, 3]. On the other hand, increased intra-abdominal pressure and ground-reaction forces may also stimulate the contraction of pelvic floor muscles (PFMs).

\section{Anatomical and functional concerns}

Pelvic floor muscles have two important roles; they serve as the support for abdominal organs and are crucial for closure of the urethra, vagina and rectum. Their anatomy is also subject to discussion; thus some structures (muscles, ligaments, fascias) are not always anatomically recognized. Aside from their functional role, some of them may even be hypothetical (e.g. does the fascia pelvis exist?). Main muscles include: levator ani, coccygeus, obturator internus and the piriformis muscle. The levator ani is cone-shaped, embraces the vagina and consists mostly of transverse striated muscle, but some smooth muscles have also been reported [10-12]. This indicates its major role under short-term strain and its connection to the urethra is important in stabilization and prevention of damage [12]. The true anatomical connection between the levator ani and the urethral sphincter is also subject to discussion as to whether it really exists [12]. Even if an anatomical connection is dubious, the PF muscles should be considered as a "complex", with the urethra being crucial for urine continence.
The puborectalis muscle as a part of the levator ani muscle is the most important for urine continence. The fast muscle fibers respond quickly, and partially involuntarily, when the intraabdominal pressure increases [13]. Some authors also distinguish the pubococcygeus muscle, although magnetic resonance imaging (MRI) findings suggest its role is rather more supportive, whereas the puborectalis muscle has a strong urethral closure function. The whole mechanism of the PFM is described as changes between "basin" to "dome" shape. The contracting pubococcygeus and iliococcygeus muscles shift the coccygeus ventrally and cranially $[10,11]$. Down to the pelvic floor the genitourinary diaphragm is perforated by the urethra and vagina. The transverse perineal muscle embraces the urethra, forming the sphincter. Other muscles have been identified, but their anatomical presence has also been questioned during surgical procedures $[14,15]$. The inner urethral sphincter consists of smooth muscle continuing from the detrusor muscle. Both are controlled involuntarily. The outer sphincter consists of transverse striated muscle and is voluntarily controlled. The female urethral sphincter also consists of a compressor urethral muscle and a urethrovaginal sphincter $[16,17]$. Bardoni et al. described both the thoracic diaphragm and pelvic floor diaphragm as functionally similar. When contracted they elevate, and during relaxation they descend. Their constant cooperation is important in regulating intra-abdominal pressure. In sportswomen this is even more important due to respiratory demands and the effort required from core muscles. The pelvic floor is anatomically connected to the transverse abdominal muscle, thoracolumbar fascia and gluteus muscle [18]. The stability of pelvic support determines urine continence [19].

Urinary continence is determined by the balance between intravesical and intraurethral pressure. Intravesical pressure is also influenced by intraabdominal pressure, whereby intraurethral pressure is controlled by the sphincter and muscle "hammock" (levator ani, anterior vagina wall). When intraabdominal pressure increases, the mechanism regulating the closure of the urethra consists of shortening the sub-urethral part of the vagina and levator ani, with simultaneous sacrouterine ligaments that pull in a posterior direction. If the proper reflex and balance or the posture is dysregulated, it can often lead to UI in young athletes. This could be exacerbated by an excess of superficial muscle training without the precaution of deep muscle training (transverse and oblique). Urinary incontinence is a result of a disbalance between pushing pressure (intraabdominal transmitted to central perineal ligaments and sacrococcygeal ligaments) and resisting pressure 
(pelvic floor and pelvic organs) [20]. As a result of some hypoestrogenism (loss of menstrual periods, loss of fatty tissue) younger athletes are especially at risk. Other risks for younger athletes are: lumbar hyperlordosis due to a lack of proper abdominal musculature training, which tends to horizontal sacral bone, promoting different pelvic angles [20].

During physical activity the rectus abdominis muscle and oblique abdominal muscles stabilize the lumbar-pelvic-iliac complex and additionally increase the intra-abdominal pressure [21, 22]. The second group of this complex consists of transverse abdominal muscle (TrA), the lumbar multifidus muscle, thoracic diaphragm and the PFM. During activity the TrA acts as a powerful stabilizer with limited movement. But its role in increasing intra-abdominal pressure also translates into the displacement of inner organs towards the pelvic floor. Proper cooperation between this complex and the PFM is needed [21]. With aging athletes the muscle is substituted by fatty tissue that destabilizes the lumbar-pelviciliac complex with a change in intra-abdominal pressure [23]. Both the thoracic diaphragm and the PFM consist of fast and slow contractile muscles. When acting properly they determine the stabilization of the pelvic floor during sport activities. The intraurethral pressure should always exceed the intra-abdominal pressure, and in our opinion the muscle timing is the most important. Junginger et al. proved that only simultaneous contraction of the TrA and PFM provides urine continence [24]. We would even suggest that prior PFM contraction to TrA is the best pattern for female athletes. In a pathological condition TrA activation prior to PFM provokes urine leakage. This becomes more pronounced during high impact activity, and improper muscle contraction timing is more important than PFM strength itself. According to Thomson et al., and also in our opinion, the proper vector of the PFM contraction pattern is the second mechanism in continence of urine. Physiologically, as seen in ultrasound examination, the bladder neck elevates during PFM contraction. In dysfunction the bladder neck descends or does not move, and this could be enhanced by intra-abdominal pressure $[25,26]$. The caudal vector of the PFM excludes the proper closure of the urethra and is more frequently seen in athletes $[27,28]$. Interestingly, submaximal contraction of the TrA and PFM enables the elevation of the bladder neck, but additional activation of internal oblique muscles makes it impossible [24]. Thus, we suggest making an evaluation of the oblique muscle in ultrasound during exercise. The previously described mechanisms, important for athletes during high impact training, did not include urethral movement.
A weak PFM does not support the urethra, and this is a condition that can also be described in ultrasound [29-33]. During the Valsalva maneuver in some women the urethra remains in the same location and is not displaced by the pubovaginalis or puborectalis muscle, and the urethral closure is ineffective. This mechanism could be more frequent in young athletes where no PFM deficiency is observed. It suggests that proper urine continence is conditioned by proper muscle elasticity and dynamics rather than the power of contraction. $B \varnothing$ hypothesizes that in athletes repeated intra-abdominal pressure peaks create caudal force on the PFM, implicating muscle weakness [34]. After years of training the intraurethral closure pressure is not sufficient, as proven by Hagovska et al. [35]. Capson et al. observed in electromyography that during the Valsalva maneuver the contraction power of the PFM was higher in the vertebral neutral position and lower in athletes with excessive or low lumbar lordosis [36].

The anatomical structure of the pelvic floor muscles determines their function, which can be improved through appropriate exercise. Professional physiotherapy takes into account the phasic and tonic work of the pelvic floor muscles. The first is practiced with a short contraction time and longer relaxation, and the second with a long contraction time and the same relaxation time. The number of tensions is adjusted individually to the patient's clinical condition. Several authors have concluded that quality is better than quantity with regard to contraction of the pelvic floor muscles [37].

\section{How does the functional anatomy of the pelvic floor translate into clinical practice in athletes?}

A study among Portuguese athletes compared to controls revealed interesting data. Elite athletes were divided into groups of different sport disciplines with respect to training and exercise characteristics. They all had lower parity, BMI, less constipation or a family history of UI. The highest prevalence of UI was among gravity sports (84.4\%); for other groups of sports, including technical sports, endurance sports, power sports and weight sports, prevalence of this condition was reported between $14.3 \%$ and $33.3 \%$. Most of the symptoms occurred during practice or at the end of training or a competition. Most of the women use a pad to reduce urine leakage. They also consider $\mathrm{UI}$ as a factor which affects their performance [2]. Among cyclists and soccer players incontinence urge is more apparent [4]. High intensity training and competition measured by MET-min ( $>$ 8.0) could also increase the risk for SUI among young women (<35) in various types of sport. The highest risk 
was found for ball sports and athletics [3]. The incidence among nulliparous athletes is correlated directly with age and years and intensity of training. This condition remains hidden in younger athletes and can be one of the reasons for retiring from a sport [20]. The incidence of SUI is strongly associated with training volume and sport rankings in a dose-dependent manner [6]. Medically there is also a negative association of $\mathrm{UI}$ induced infections, ulcers and sleep disorders with sport performance [4]. In some disciplines, such as cheerleaders, fecal incontinence is more common than $\mathrm{UI}$, and is reported by as many as $26.9 \%$. No major differences were noted when dividing this group into bases and flyers [38].

Exercising women with UI may leak during physical activity while being continent during normal physical activity. However, it is often the case that top athletes are also at a higher risk of $\mathrm{UI}$ during daily activities, particularly those who practice gravity sports [4]. A very interesting observation was made by Thyssen et al., namely that athletes experience more leakage during training than in competition ( $95 \%$ vs. $51 \%$ ). This is probably due to high catecholamine levels during competition; they act as $\alpha$-receptors maintaining urethral closure [39]. Women triathletes form a special group. While they are younger than the typical population or elite sportswomen, they have lower prevalence of risk factors for UI/SUI. The controls show a higher prevalence of risk factors typically considered as increasing the risk of UI. On the other hand, as amateur triathletes become older they may accumulate more life risk factors such as deliveries, constipation or chronic disease. The high-impact sport itself does not seem to affect child delivery; thus obstetrical history is less important in urinary incontinence among sportswomen than strenuous exercise $[40,41]$. There is no coherent literature in this field, although general factors are known and differences could be clinically irrelevant when considering triathlons. In the literature there is little in-depth analysis of the pathology or distinct features, and guidelines are lacking. However, we can expect that with the growing demand for quality of life and the growth of elite and amateur sports, the health service will have to address the problem of how to treat younger women with UI.

There are two opposing theories about how physical activity may affect the pelvic floor: (1) physical activity strengthens the PFMs; (2) exercises overload and weaken the pelvic floor. However, neither of them has been proved to date [34]. Athletes report more intense leakage at the end of training or competition. This suggests that they suffer from a lack of muscular endurance of the pelvic floor. Being a former elite athlete is probably not associated with later UI risk, but when performing sport $\mathrm{UI}$ is strongly associated with persistent urinary incontinence in future [4]. It is also uncertain whether high-impact sport causes a PFM imbalance with abdominal muscle fatigue or whether it damages the PFMs and leads to SUI. This question should be answered in well-designed studies. The more frequent the impact associated with increased intra-abdominal pressure, the greater should be the restraint and support of PFMs, which must be strengthened to prevent SUI and preserve the function [6, 42]. Moreover, eating disorders, including sport anorexia, among athletes such as long-distance runners can be associated with UI [4].

Participation in high impact and long-lasting training can increase the thickness of the levator ani and puborectalis without a direct influence on sub-urethral mechanical support and UI prevalence. When measured by MRI and ultrasound, women athletes were found to have increased muscle diameter, greater bladder neck descent and a larger hiatal area during the Valsalva maneuver. Greater muscle thickness can compensate for higher muscle distensibility [40]. In another study, volleyball and basketball athletes were found to exert lower perineal contracting pressures than non-athletes, thus being more susceptible to a higher risk of UI. There was also a strong correlation between lower perineal pressure and training volume. This suggests that there is a lack of PFM co-training in these disciplines [42]. However, observations based on the limited volume of studies may be misleading because athletes with thicker PFMs could simply continue their high-impact training and could have a better peri-urethral collagen type I to III ratio. Hypermobility of the urethra, which is typical for a decreased type I/III collagen ratio, is associated with pelvic organ prolapse prevalence rather than with UI. Nevertheless, adding occult SUI due to hypermobility of the kinking urethra may reveal that the true prevalence of urine leakage exceeds pelvic organ prolapse [40, 42].

\section{Diagnostics and treatment (training, co-training or scalpel?)}

Stress urinary incontinence among sportswomen should be interdisciplinarily treated by physicians and physiotherapists with a knowledge of functional diagnostics. Previously, other approaches have been adopted with controversial results. Although bladder training can have a positive outcome in some cases of overloading, female athletes entering training or competition generally empty their bladder. Electrostimulation in the general population has also yielded questionable results [34]. More recent research indicates the advantage of high-intensity focused electromagnetic 
(HIFEM) therapy over electrostimulation. Ultrasound has shown positive changes in the posttreatment dynamics of the pelvic floor (decreased anteroposterior diameter, laterolateral diameter, and hiatal area) [43]. Recently, a randomized trial showed that PFM training among athletes produced positive results [44]. Early postpartum exercising has not been found to have a negative influence on SUI, so even athletes at an elite level should not be discouraged from training [45]. We also recommend that both professions, physicians and physiotherapists, should use pelvic floor sonography (PFS) as an objective tool for diagnosis, prognosis and treatment monitoring. Initial good instruction for a patient on how to contract the PFM is crucial. According to the literature, half of women are unable to distinguish the PFM from abdominal muscle contractions. Vaginal examination during contraction is discussed by Mateus-Vasconcelos et al., but no standardization has ever been made. Recently, the digital vaginal palpation method has been proposed for PFM contraction evaluation along with proper feedback for the patient to increase its therapeutic value. We suggest that the effects in PFS should be periodically checked. The hypopressive and relaxing method will promote intraabdominal pressure more towards the thoracic diaphragm, thereby sparing the pelvic floor, the so-called "Monica Seles cry" [20]. Hypopressive exercises based on diaphragm relaxation and a decrease of intraabdominal pressure should accompany PFM training [46]. The physiotherapy should include: proper vertebral balance, unblocking of the thoracic diaphragm, and the normotonic abdominal wall (neither hyper- nor hypotonic) [20]. Bernardes et al. propose hypopressive exercises during PFM contractions in the horizontal and vertical position (10 times 3-8 s) [47]. McLean et al. also observed that after PFM training ( $3 \times 12$ contractions), the proper pattern is advantageous in comparison to the contraction power itself [48]. There are few publications, but we also suggest including gynecologic pelvic floor sonography for teaching proper muscle contraction [30-33]. Easy sonographical access to these structures, with the possibility to present them dynamically in real time to the patient, is an ideal feedback method. It is crucial for younger athletes with a short urethra or a hypermobile kinking urethra because this condition could implicate a failure of incontinence prevention or PFM training. Wen et al. described urethral hypermobility in an ultrasound examination in SUI, mobility being over $20 \mathrm{~mm}$ and a change in the urethral kinking angle during the Valsalva maneuver $[46,49]$. Junginger et al. examined the bladder neck with ultrasound and electromyography from the vagina and abdominal wall. They concluded that the proper pattern of PFM contraction is the most important without engagement of the abdominal muscles [24]. Iha et al. treated women with SUI according to NICE (National Institute of Clinical Excellence) with and without electrostimulation ( $3 \times 8$ contractions daily). Some improvement in sexual function was noted, but the results remain controversial in the intervention group. This is probably due to insufficient contractions and a lack of individualized muscle endurance estimated by functional diagnostics [50]. In other studies 8-12 contractions lasting for $10 \mathrm{~s}$ several times daily were more effective [51]. Luginbuehl et al. described PFM training with involuntary contractions in SUI women which was more effective in continence. This observation is relevant to sportswomen and in daily activity during involuntary intra-abdominal pressure increase. Sport training should also include pelvic floor neuro-muscular preparation for unexpected abdominal contractions [52]. During the muscle contraction the pelvic floor should lead to ventral and cranial movement and during the Valsalva maneuver the perineum should not move down without abdominal contraction [53]. According to our experience in urogynecological physiotherapy the caudal movement is acceptable only when previous cranial contraction of the pelvic floor is present. This contraction ahead of abdominal pressure increase is crucial for PFM stabilization in preventing incontinence.

Routine examinations of SUI should include vaginal atrophy and pelvic organ prolapse (POP) assessment, as well as other tests, such as pad and urodynamic examination, in unclear or complex cases. In our opinion, routine examinations of SUI should include a functional test with a full bladder, such as the cough test and PFS, as the only objective and most important urogynecological tool. Behavioral aspects of incontinence should also be discussed or surveyed by questionnaires to fully understand miction disturbance in women.

Ultrasound examination is more often used for diagnosing urogynecology patients. An advantage of ultrasound is the possibility of multiple repetitions in a short time with close contact with the patient and a real-time evaluation. The transvaginal probe has a high resolution, but a short range, although it is routinely used in gynecology. Ambulatory ultrasound scanning is important in gynecologic primary care worldwide. However, ultrasound with a vaginal probe for SUI is sufficient to visualize the urethra and the bladder neck. We would suggest that 2D ultrasound performed with a transvaginal probe (PFS-TV) be used in urogynecology centers more often, because many specialists are familiar with a transvaginal probe, which offers real-time high quality images with 
a minimal influence on the female pelvic floor. The examination lasts additional minutes, but the advantages in diagnostics, functional diagnostics and contraction feedback are discussed in other parts of the article. In our opinion, using a small transvaginal probe outside the vagina minimizes the influence of the probe on the pelvic floor changes that occur during the Valsalva maneuver or pelvic floor muscle exercises. A detailed conception of PFS-TV is presented in Figure 1 [30-33].

Functional diagnostics should initially qualify women athletes for physiotherapy. The goal of the physical therapy is proper PFM function education. The techniques include manual therapy of the lumbo-pelvic-iliac complex and pelvic floor muscle training in real time with biofeedback (tonic and phase contractions). This can be achieved under ultrasound or electromyography. In ultrasound we can diagnose and teach cases of improper PFM contraction patterns (no movement or depression) and urethra movement. However, during real sport activity this method is limited [30-33]. Electromyography is more useful in evaluating PFM together with abdominal muscles during sport activities. It also enables the measurement of contraction power and timing, and can evaluate progress in urogynecology treatment. In the first training phase all exercises should be performed in a horizontal position with flexed lower extremities. In the subsequent phases modification of the position of the pelvis is possible and additional activities should be introduced. The number of PFM contractions should always be individualized for every woman athlete.

To assess the problem the pad test can quantify urine loss objectively, if the apparent incontinence is present. However, the pad test can quantify, but not reveal, the occult urinary incontinence. Different protocols have been described in the literature, but the most common protocols recommended by the International Continence Society are the 1-hour and 24-hour pad tests. This should also be performed among female athletes, especially because their quality of life and the demands of their sport are higher than in non-athletes. However, it could also disguise the true quantification of the problem and thus lead to surgical overtreatment. It is reasonable to perform both tests to check whether urine loss is occasional during exercise and absent in normal activities [5].

The treatment options for younger athletes, when a muscular disbalance is possible, require an individualized approach. Pharmacotherapy cannot treat anatomical factors such as urethral support, vesical neck function, and function of the PFM. Some drugs influencing urethral resting pressure

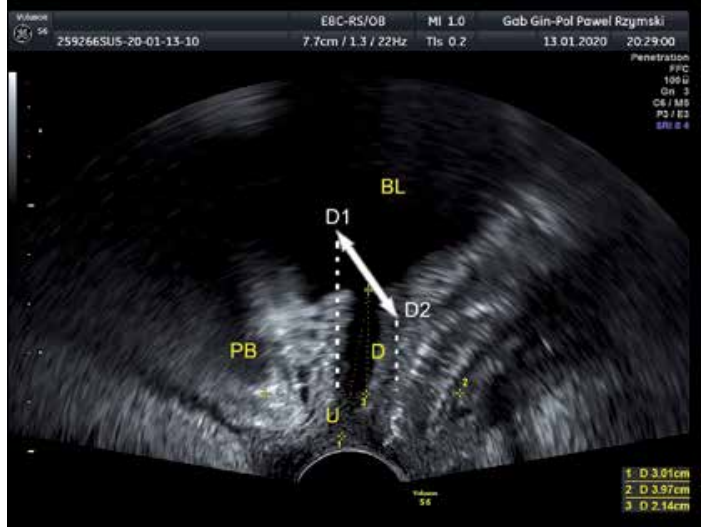

Figure 1. Pelvic floor sonography with a transvaginal probe (PFS-TV), U - urethra, BL - bladder, PB symphysis pubis, $D$ - elevation over reference line (lower margin of symphysis pubis). During the examination the physiology of urethral movement is followed: during urethral closure (D1) and during pushing (D2) with intraabdominal pressure

previously researched in SUI include $\alpha$-adrenoceptor agonists (ephedrine, norephedrine, cones, phenylpropanolamine), imipramine, clenbuterol, duloxetine and estrogen, with controversial results, few randomized controlled trials and none among athletes [34]. We can consider oestrogens when hypoestrogenism is present or even routinely in postmenopausal athletes, especially because of the higher risk of developing osteoporosis. After deep analysis of risk factors, such as eating disorders, hypoestrogenism, caffeine and alcohol intake, precise fluid intake should be discussed. Athletes should void shortly before training, but the fluid intake should cover their hydration needs [39]. Pelvic floor training is crucial for both prophylaxis and treatment of urinary incontinence. It should be considered as a first line treatment together with mechanical support. Especially valuable methods for athletes are pessaries and tampons, as they are the only non-invasive options that offer proper urethral support during longer physical activity. The only report of athletes using tampons during activity showed total dryness during activity $[34,39]$. Long-term compliance of pessaries in SUI is affected by age, with better compliance in those over 60. No such studies have been conducted among athletes, but we suppose that avoidance of surgery and intermittent (occasional) problems with SUI should implicate good long-term athletes' compliance [54].

Pharmacological treatment has limited application in young athletes. In older sportswomen the prevalence of urge or mixed incontinence could be higher, but still hypermobile urethra or occult incontinence with urge should be considered. The failures in proper incontinence treatment can be attributed to: wrong diagnosis with 


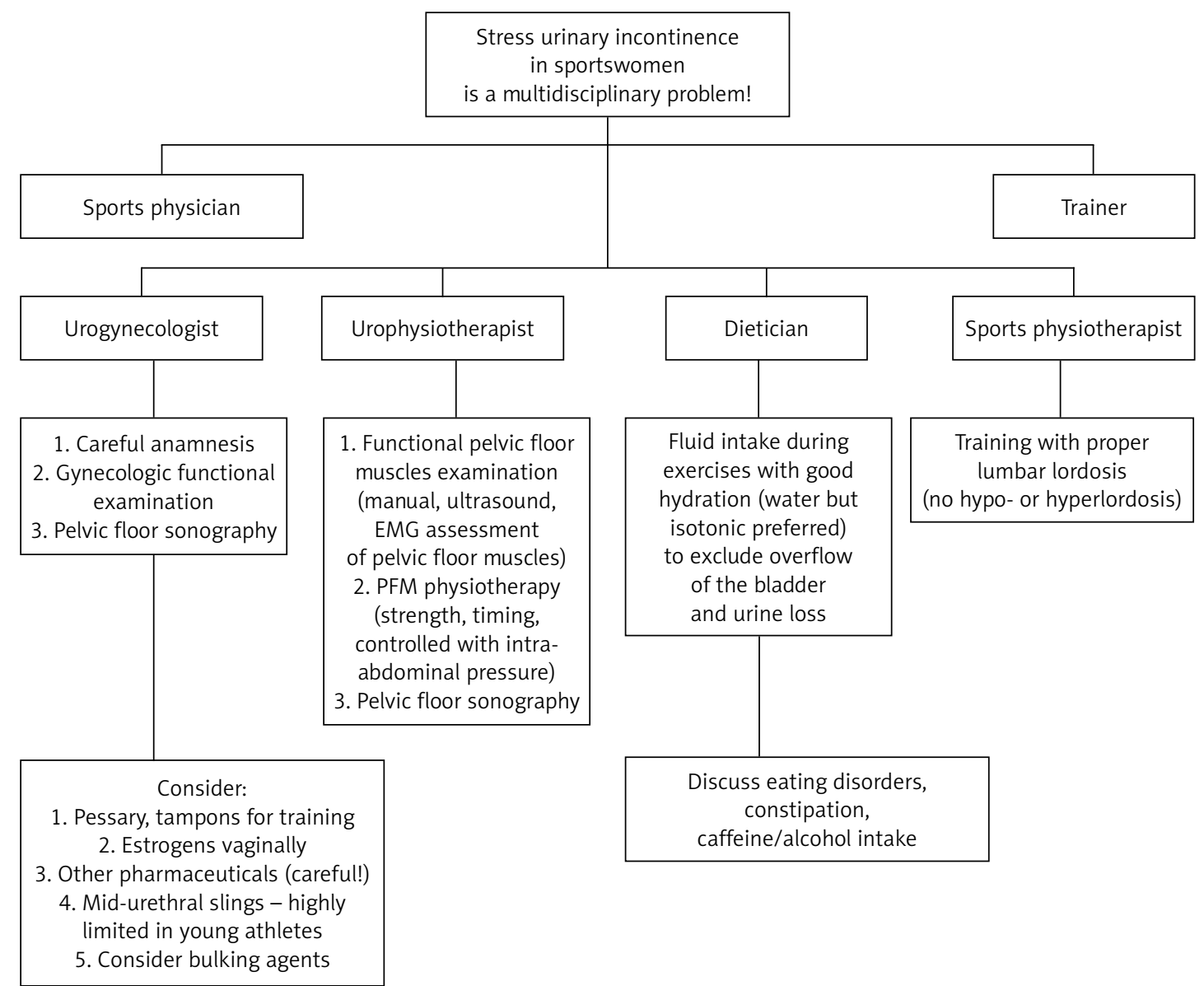

Figure 2. Interdisciplinary treatment of stress urinary incontinence in sportswomen

objective pelvic floor sonography, wrong tampon/ pessary vaginal placement (too deep) or an improper physiotherapeutic approach. After clear exclusion of the above reasons among athletes with mixed or urge incontinence some pharmacological treatment should be considered. There is little literature, although duloxetine or imipramine can improve quality of life, but it is unclear whether they fully cure symptoms in athletes. Side effects are also to be expected. Pseudoephedrine hydrochloride, a common $\alpha$-adrenergic agent, is prohibited in sport when present in urine over $150 \mu / \mathrm{ml}$. Anticholinergics should be avoided in athletes because they affect the sweating mechanisms and therefore increase the risk of heat stroke [39]. If all the above options fail, surgery could be considered. However, the surgery itself may have a negative impact on the effectiveness of later PFM training, while training has no impact on future surgery. Nevertheless, urinary incontinence should be observed by the examiner and all causes carefully evaluated or excluded. Mid-urethral slings are a common method of treatment, but in young athletes are inappropriate if the leakage occurs only during sport. Among them TVT (transvaginal tape, retropubic) and TVT-O (transobturator tape) independently from the technique adopted in SUI might be similarly effective and safe at long-term follow-up. The subjective and objective cure rates varied from 61.6 to $81.3 \%$, but the studies do not separate younger women and/or athletes [55].

\section{Tips and tricks in the treatment of young women athletes (Figure 2)}

In summary, there are no randomized controlled trials or reports on the effect of any treatment for stress urinary incontinence in female athletes. However, strength and timing training of the pelvic floor muscles has been shown to be effective in SUI with a cure rate of up to 69\% [34]. In our opinion these numbers are too optimistic in athletes and should be proved in future studies. Pelvic floor muscle training has no serious adverse effects and has been recommended as a firstline treatment in the general population. The use of preventive devices such as vaginal tampons or pessaries can prevent leakage during high impact physical activity with a lack of symptoms in up to $100 \%$ of cases [56]. The pelvic floor muscles need to be much stronger in elite athletes than in other women and with an adequate contraction 
pattern, but sub-urethral support is still crucial for urine continence. Exercises to improve their function should include phasic and tonic contractions. Phase tension gives quick support for the urethra, while tonic work provides adequate stabilization of the urethra. There is no literature on bulking agents among athletes, but it is probable that future studies will provide promising results.

\section{Conflict of interest}

The authors declare no conflict of interest.

\section{References}

1. Irwin DE, Kopp ZS, Agatep B et al. Worldwide prevalence estimates of lower urinary tract symptoms, overactive bladder, urinary incontinence and bladder outlet obstruction. BJU Int 2011; 108: 1132-8.

2. Carvalheis A, Jorge RN, Bo K. Performing high-level sport is trongly associated with urinary incontinence in elite athletes: a comparative study of 372 elite female athletes and 372 controls. Br J Sport Med 2018; 52: 1586-90.

3. Hagovska M, Svihra J, Bukova A Drackova D. Prevalence and risk of sport types to stress urinary incontinence in sportswomen: a cross-sectional study. Neurourol Urodyn 2017; 37: 1957-64.

4. Goldstick O, Constantini N. Urinary incontinence in physically active women and female athletes. Br J Sport Med 2014; 48: 296-8

5. dos Santos KM, Da Rosa TH, Tonon da Luz CS, et al. Quantification of urinary loss in nulliparous athletes during 1 hour of sports training. PM R 2019; 11: 495-502.

6. Da Rosa T, Brandao S, Mascarenhas T, et al. Volume of training and the ranking level are associated with the leakage of urine in young female trampolinists. Clin J Sport Med 2015; 25: 270-5.

7. Casey EK, Temme K. Pelvic floor muscle function and urinary incontinence in the female athlete. Phys Sportmed 2017; 45: 399-407.

8. Rzymska I, Rzymski P, Wilczak M. The influence of passive and active moral training on medical university on changes of students' moral competence. Ann Agric Environ Med 2014; 21: 161-6.

9. Yi J, Tenfelde S, Tell D, et al. Triathlete risk of pelvic floor disorders, pelvic girdle pain and female athlete triad. Female Pelvic Med Recotr Surg 2016; 22: 373-6.

10. Raizada V, Mittal RK. Pelvic floor anatomy and applied physiology. Gastroenterol Clin North Am 2008; 7: 493-509.

11. Weinstein MM, Jung SA, Pretorius DH, Nager CW, den Boer DJ, Mittal RK. The reliability of puborectalis muscle measurements with 3-dimensional ultrasound imaging. Am J Obstet Gynecol 2007; 197: 68.e1-6.

12. Nyangoh Timoh K, Moszkowicz D, Zaitouna M, et al. Detailed muscular structure and neural control anatomy of the levator ani muscle: a study based on female human fetuses. Am J Obstet Gynecol 2018; 218: 121.e1-12.

13. Stoker J. Anorectal and pelvic floor anatomy. Best Pract Res Clin Gastroenterol 2009; 23: 463-75.

14. Oelrich TM. The urethral sphincter muscle in the male. Am J Anat 1980; 158: 229-46.

15. Nakajima F, Takenaka A, Uchiyama E, Hata F, Suzuki D, Murakami G. Macroscopic and histotopographic study of the deep transverse perineal muscle (musculus transversus perinei profundus) in elderly Japanese. Ann Anat 2007; 189: 65-74.
16. Oelrich TM. The striated urogenital sphincter muscle in the female. Anat Rec 1983; 205: 223-32.

17. Jung J, Ahn HK, Huh Y. Clinical and functional anatomy of the urethral sphincter. Int Neurourol J 2012; 16: 102-6.

18. Bordoni B, Sugumar K, Leslie SW. Anatomy, Abdomen and Pelvis, Pelvic Floor. StatPearls. Treasure Island (FL): StatPearls Publishing; 2019.

19. Wei JT, De Lancey JO. Functional anatomy of the pelvic floor and lower urinary tract. Clin Obstet Gynecol 2004; 47: 3-17.

20. Laffitte A. Management of urinary incontinence in the adolescent athlete. Arch Pediatr 2015; 5 Suppl 1: 196-7.

21. Richardson C, Hodges PW, Hides J (eds). Kinezyterapia w stabilizacji kompleksu lędźwiowo-miednicznego. Wrocław: Elsevier Urban \& Partner; 2009.

22. Stokes IAF, Gardner-Morse MG, Henry S. Abdominal muscle activaion increases lumbar spinal staility: analysis of contrubutions of different muscle groups. Clin Biomech (Bristol, Avon) 2011; 26: 797-803.

23. Woodham M, Woodham A, Skeate JG, Freeman M. Long-term lumbar multifidus muscle atrophy changes documented with magnetic resonance imaging: a case series. J Radiol Case Rep 2014; 8: 27-34.

24. Junginger B, Baessler K, Sapsford R, Hodges PW. Effect of abdominal and pelvic floor tasks on muscle activity, abdominal pressure and bladder neck. Int Urogynecol J 2010; 21: 69-77.

25. Thompson JA, O'Sullivan PB, Briffa NK, Neumann P. Differences in muscle activation patterns during pelvic floor muscle contraction and Valsalva maneuver. Neurourol Urodyn 2006; 25: 148-55.

26. Thompson JA, O'Sullivan PB, Briffa NK, Neumann P. Altered muscle activation patterns in symptomatic women during pelvic floor muscle contraction and Valsalva manouevre. Neurourol Urodyn 2006; 25: 268-76.

27. Jones BP, L'Heveder A, Saso S, Yazbek J, Smith JR, Dooley M. Sports gynaecology. Obstet Gynecol 2019; 21: 85-94.

28. Carvalhais A, Natal Jorge R, Bø K. Performing high-level sport is strongly associated with urinary incontinence in elite athletes: a comparative study of 372 elite female athletes and 372 controls. Br J Sports Med 2018; 52: 1586-90.

29. Pirpiris A, Shek KL, Dietz HP. Urethral mobility and urinary incontinence. Ultrasound Obstet Gynecol 2010; 36: $507-11$

30. Wlaźlak E, Kluz T, Surkont G, Kociszewski J. Urethral funneling visualized during pelvic floor sonography - analysis of occurrence among urogynecological patients. Ginekol Pol 2018; 89: 55-61.

31. Dresler MM, Kociszewski J, Wlaźlak E, Pędraszewski P, Trzeciak A, Surkont G. Repeatability and reproducibility of measurements of the suburethral tape location obtained in pelvic floor ultrasound performed with a transvaginal probe. J Ultrason 2017; 17: 101-5.

32. Wlaźlak E, Kluz T, Kociszewski J, et al. The analysis of repeatability and reproducibility of bladder neck mobility measurements obtained during pelvic floor sonography performed introitally with 2D transvaginal probe. Ginekol Pol 2017; 88: 360-5.

33. Wlaźlak E, Kociszewski J, Suzin J, Dresler M, Surkont G. Urethral length measurement in women during sonographic urethrocystography - an analysis of repeatability and reproducibility. J Ultrason 2016; 16: 25-31.

34. $B \varnothing K$. Urinary incontinence, pelvic floor dysfunction, exercise and sport. Sports Med 2004; 34: 451-64.

35. Hagovska M, Švihra J, Buková A, Dračková D, Švihrová V. Prevalence and risk of sport types to stress urinary in- 
continence in sportswomen: a cross-sectional study. Neurourol Urodyn 2018; 37: 1957-64.

36. Capson AC, Nashed J, Mclean L. The role of lumbopelvic posture in pelvic floor muscle activation in continent women. J Electromyogr Kinesiol 2011; 21: 166-77.

37. Ramirez Garcia I, Ratto LB, Kauffmann S, et al. Rehabilitación del suelo pélvico femenino. Bogota: Panamericana; 2013, p. 200.

38. Carvalho C, da Silva Serrao PRM, Beleza ACS, et al. Pelvic floor dysfunction in female cheerleaders: a cross-sectional study. Int Urogynecol J 2020; 31: 999-1006.

39. Thyssen $\mathrm{HH}$, Clevin L, Olesen S, et al. Urinary incontinence in elite female athletes and dancers. Int Urogynecol J 2002; 34: 451-64.

40. Kruger JA, Dietz HP, Murphy BA. Pelvic floor function in elite nulliparous athletes. Ulstrasound Obstet Gynecol 2017; 30: 81-5.

41. Sigurdardottir T, Steingrimsdottir T, Geirsson RT, et al. Do female athletes experience more complicated childbirth than non-athletes? A case-control study. Br J Sport Med 2019; 53: 354-8.

42. da Silva Borin LCM, Nunes FR, de Oliveira Guirro EC. Assessment of pelvic floor muscle pressure in female athletes. Phys Med Rehab 2013; 5: 189-93.

43. Silantyeva E, Zarkovic D, Astafeva E, et al. A comparative study on the effects of high-intensity focused electromagnetic technology and electrostimulation for the treatment of pelvic floor muscles and urinary incontinence in parous women: analysis of posttreatment data. Female Pelvic Med Reconstr Surg 2020. doi: 10.1097/SPV.0000000000000807

44. Pires TF, Pires PM, Moreira MH, et al. Pelvic floor muscle training in female athletes: a randomized controlled pilot study. Int J Sports Med 2020; 41: 264-70.

45. Tennfjord MK, Engh ME, Bø K. The influence of early exercise postpartum on pelvic floor muscle function and prevalence of pelvic floor dysfunction 12 months postpartum. Phys Ther 2020; 100: 1681-9.

46. Mateus-Vasconcelos ECL, Ribeiro AM, Antônio Fl, Brito LGO, Ferreira $\mathrm{CHJ}$. Physiotherapy methods to facilitate pelvic floor muscle contraction: a systematic review. Physiother Theory Pract 2018; 34: 420-32.

47. Bernardes BT, Resende AP, Stüpp L, et al. Efficacy of pelvic floor muscle training and hypopressive exercises for treating pelvic organ prolapse in women: randomized controlled trial. Sao Paulo Med J 2012; 130: 5-9.

48. McLean L, Varette K, Gentilcore-Saulnier E, Harvey MA, Baker K, Sauerbrei E. Pelvic floor muscle training in women with stress urinary incontinence causes hypertrophy of the urethral sphincters and reduces bladder neck mobility during coughing. Neurourol Urodyn 2013; 32: 1096-102.

49. Wen L, Shek KL, Dietz HP. Changes in urethral mobility and configuration after prolapse repair. Ultrasound Obstet Gynecol 2019; 53: 124-8.

50. Jha S, Walters SJ, Bortolami O, Dixon S, Alshreef A. Impact of pelvic floor muscle training on sexual function of women with urinary incontinence and a comparison of electrical stimulation versus standard treatment (IPSU trial): a randomised controlled trial. Physiotherapy 2018; 104: 91-7.

51. de Vries AM, Heesakkers JPFA. Contemporary diagnostics and treatment options for female stress urinary incontinence. Asian J Urol 2018; 5: 141-8.

52. Luginbuehl H, Lehmann C, Baeyens JP, Kuhn A, Radlinger L. Involuntary reflexive pelvic floor muscle training in addition to standard training versus standard train- ing alone for women with stress urinary incontinence: study protocol for a randomized controlled trial. Trials 2015; 16: 524

53. Messelink B, Benson T, Berghmans B, et al. Standardization of terminology of pelvic floor muscle function and dysfunction: report from the pelvic floor clinical as sessment group of the International Continence Society. Neurourol Urodyn 2005; 24: 374-80.

54. Hsieh MF, Tsai HW, Liou WS, et al. Long-term compliance of vaginal pessaries: does stress urinary incontinence matter? Medicine (Baltimore) 2019; 98: e15063.

55. Maggiore ULR, Agro EF, Soligo M, Li Marzi V, Digesu A, Serati M. Long-term outcomes of TOT and TVT procedures for the treatment of female stress urinary incontinence: a systemic review and meta-analysis. Int Urogynecol J 2017; 28: 1119-30.

56. Glavind K. Use of a vaginal sponge during aerobic exercises in patients with stress urinary incontinence. Int Urogynaecol J Pelvic Floor Dysfunct 1997; 8: 351-3. 